\title{
Solid state battery formation of triglycine sulphate and triglycine sulphate selenate
}

\author{
S H CHAVAN \\ Department of Physics, Shivaji University, Kolhapur 416 004, India \\ MS received 5 April 1988; revised 4 July 1988
}

\begin{abstract}
The d.c. electric field effect on ferroelectric triglycine sulphate (TGS) and TGSSe crystals at high temperature above the Curie temperature shows the formation of a solid state battery. The current and the electromotive force of the battery are measured and their changes observed near the Curie temperature. Even if the electrodes are shortcircuited the emf recovers almost to the same value.
\end{abstract}

Keywords. Ferroelectrics; solid state battery; Curie temperature; hysteresis.

\section{Introduction}

The formation of solid state battery (SSB) by ferroelectric materials is interesting and has promising applications (Sawada et al 1958, 1961). Ishibashi et al (1969) who studied the electric field effect on ferroelectricity of $\mathrm{KNO}_{3}$ crystals observed an electromotive force (emf) of about $1 \mathrm{~V}$ when a d.c. electric field of about $1 \mathrm{kV} / \mathrm{cm}$ was applied to the crystal at high temperature. The emf magnitude with polarity similar to that of applied d.c. voltage is temperature-dependent. It disappears at a low temperature but recovers when the samples are heated again. EMF decays with time but the decay constant is quite large, while the time constant of the condenser composed of $\mathrm{KNO}_{3}$ and electrodes was estimated as $<10^{-8} \mathrm{~s}$. Even after the electrodes were short-circuited the emf gradually recovers to almost the same voltage without any short-circuitary.

Chavan and Kulkarni (1985a, b) and Suryawanshi and Chavan (1987) showed the formation of a solid state battery of solid solutions $\left(\mathrm{Ba}_{x} \mathrm{Sr}_{1-x}\right) \mathrm{TiO}_{3}$ and $\left(\mathrm{Ba}_{x} \mathrm{Ca}_{1-x}\right)$ $\mathrm{TiO}_{3}$ (lead-strontium) titanate and (lead-calcium) titanate, sodium vanadate and potassium vanadate respectively. This paper presents the formation of SSB of ferroelectric crystals triglycine sulphate (TGS) and triglycine sulphate selenate (TGSSe).

\section{Experimental}

Large crystals of TGS and TGS-Se were grown by reacting an aqueous glycine solution with a corresponding sulphuric/selenic acid. The growth was achieved by slowly lowering the temperature at a constant supersaturation. The crystals thus obtained could be easily cleaved on $(010)$.

A solid state battery was formed using TGS and TGS-Se single crystal plates with faces perpendicular to the ferroelectric axes. Crystal formation was confirmed by X-ray diffractometer.

The experimental set-up consisted of a globar furnace and an electronically regulated power supply to provide d.c. electric field, a digital voltmeter, a microampere current meter (VMV15) and a temperature controller arrangement. 
The thickness of the crystal plates was $0.12 \mathrm{~cm}$ (TGS) and $0.11 \mathrm{~cm}$ (TGS-Sc). A silver paste was used for good electrical contact. The electrodes were of stainless steel. A d.c. electric field of about $1 \mathrm{kV} / \mathrm{cm}$ was applied to the plates at a high temperature and then taken off. The plates were fused and the d.c. electric field taken out. Immediately thereafter the emf and the current produced were measured.

It was observed that the emf decayed slowly with time and the decay constant was high. Usually at room temperature the emf observed was small but when a drop of water was added the emf across the electrodes increased.

\section{Results and discussion}

The emf generated across the fused samples of TGS and TGS-Se is shown in figures 1 and 2 respectively. The current temperature variations through the samples are shown in figures 3 and 4 for TGS and TGS-Se respectively. It is clear from these figures that both the generated emf and the current depend upon temperature. Drastic changes in emf and current were observed at $49^{\circ} \mathrm{C}$ and $35^{\circ} \mathrm{C}$ for TGS and TGS-Se respectively (TGS and TGS-Se are in 1:1 molar proportion). These temperatures correspond to phase transitions of the respective samples. We have determined the transition temperatures of the above mentioned solid solutions by using the circuit of Sawyer and Tower (1930), wherein the hysteresis loop disappears at the transition temperature. The emf and current disappear at low temperature but reappear on heating. This could be explained as follows.

When a d.c. electric field is applied across the sample of TGS at a high temperature, part of it gets decomposed into $\mathrm{NH}_{3}^{+} \mathrm{CH}_{2} \mathrm{COON}$ and $\mathrm{O}_{2}$ ions, while $\mathrm{SO}_{2}$ remains as the residue. $\mathrm{O}_{2}$ escapes from the positive terminal as a gas while $\left(\mathrm{NH}_{3}^{+} \mathrm{CH}_{2} \mathrm{COOH}\right)$ remains near the negative terminal. Therefore, we obtain the battery of

steel, $\mathrm{NH}_{3}^{+} \mathrm{CHCOOH} /\left(\mathrm{NH}_{2} \mathrm{CH}_{2} \mathrm{COOH}\right)_{3} \mathrm{H}_{2} \mathrm{SO}_{4} / \mathrm{SO}_{2}, \mathrm{O}_{2}$, steel.

A similar reaction probably takes place in TGS-Se crystal. Usually at room temperature no large emf and current are observed. If a drop of water is added to the sample, the emf will soon appear. This is probably because hydroxide is formed and $\mathrm{H}_{2}$ gas is liberated due to the addition of water. $\mathrm{H}_{2}$ might be at the negative terminal and $\mathrm{O}_{2}$ at the positive terminal.

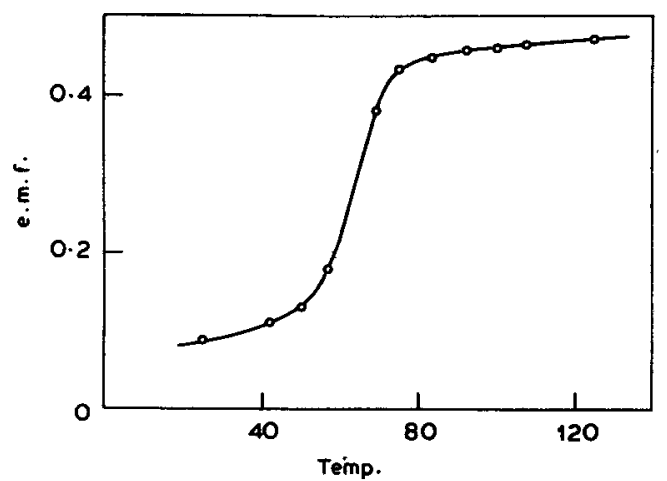

Figure 1. EMF observed in the TGS fused sample at different temperatures. 


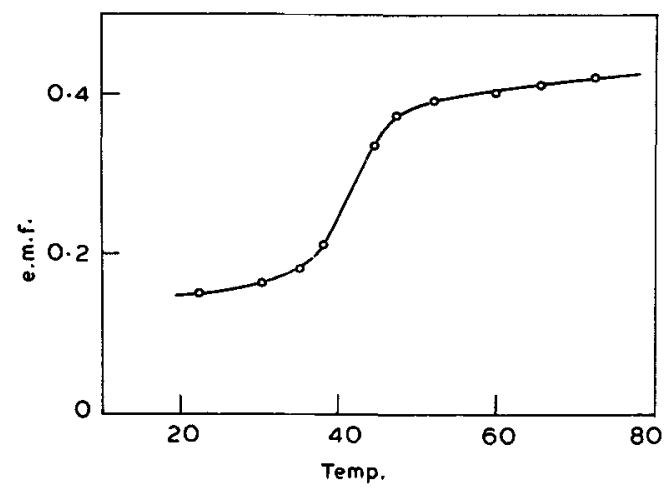

Figure 2. EMF observed in the TGS-Se fused sample at different temperatures.

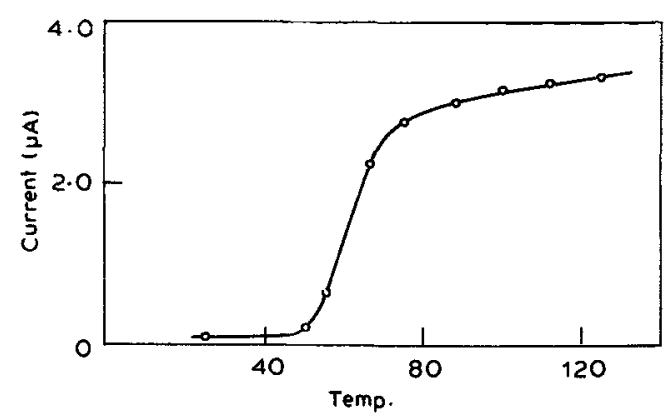

Figure 3. Current observed in the TGS fused sample at different temperatures.

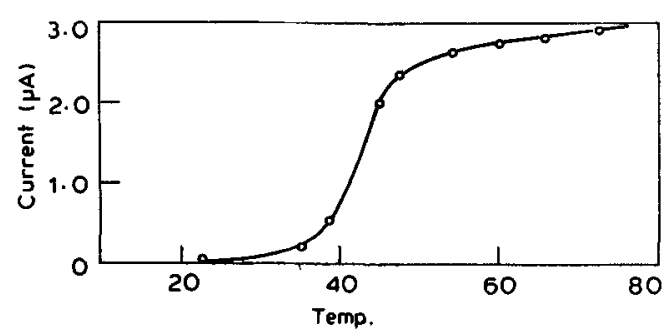

Figure 4. Current observed in the TGS-Se fused sample at different temperatures.

The present study reveals the following results: (i) Current and emf increase with temperature and a drastic change in emf and current appears at the Curie temperature. (ii) At high temperature the effect of d.c. electric field on TGS and TGS-Se shows that SSB is formed. (iii) The response of SSB is satisfactory above the Curie temperature. (iv) The ferroelectric material shows a sudden appearance of surface charge at the transition, but when electrodes are short-circuited the emf gradually recovers to almost the same value as could be expected without shortcircuitory. Since SSB is formed for TGS and TGS-Se by the application of d.c. electric-field, it will be difficult or impossible to study its effect on the ferroelectricity 
of these crystals, such as shift of ferroelectric phase transition temperature and change of dielectric constant due to the d.c. electric field.

\section{References}

Chavan S H and Kulkarni P G 1985a Indian J. Pure Appl. Phys. 23531

Chavan S H and Kulkarni U N 1985b Indian J. Pure Appl. Phys. 23533

Ishibashi Y, Midorikwa M and Takagi Y 1969 Jpn. J. Appl. Phys. 8812

Sawada S, Nomura S and Asao Y 1961 J. Phys. Soc. Jpn. 162486

Sawada S, Nomura S and Fuji S 1958 J. Phys. Soc. Jpn. 131549

Sawyer C B and Tower C H 1930 Phys. Rev. 35269

Suryavanshi S G and Chavan S H 1987 Indian J. Phys. A61 162 